\title{
STUDY ON THE PHOTO-BASED 3D SCANNING PROCESS
}

\author{
Álmos KRISTÁlY*1 AND PÉTER FICZERE ${ }^{1}$ \\ ${ }^{1}$ Department of Railway Vehicles and Vehicle System Analysis, Budapest University of Technology and \\ Economics, Múegyetem rkp. 3, Budapest, 1111, HUNGARY
}

\begin{abstract}
The experiment aims to determine the practical applicability of photo-based 3D scanning technologies. With the help of computer software, 3D printable, simulation-ready models are created from regular photographs. The accuracy and consistency of such models are evaluated regarding usability engineering.
\end{abstract}

Keywords: 3D modelling, 3D scanning, photo-based 3D models

\section{Introduction}

With the development of manufacturing and modelling technologies, more and more complex geometries can be created. At the beginning of the 20th century, point-perfect shop drawings were used for production. Nowadays, computer numerical control (CNC) is used exclusively for the control of machine tools worldwide. CNC codes can be generated directly from digital 3D geometries (CAD models, Fig. 1) and immediately sent to the machine tool via online connected systems.

CAD models are created based on planned or measured dimensions. Although this method is faster than handmade shop drawings, a complex CAD model can still take months to create from scratch.

A more straightforward method is reverse engineering, during which all data necessary for simulation or manufacturing is documented from an already existing part [1]. Planning is unnecessary; for the purpose of creating the CAD model, it is sufficient to measure the part. Difficulties can occur in the case of spherical, parabolic or other irregular surfaces that are hard to measure.

3D scanning technologies substantially simplify this process. Since the 1960s, numerous methods have been invented [2]. At first, contact probes were used to measure each point of a surface, which were then saved as coordinates with regard to the machine coordinate system. Later on, optical methods were developed. By photographing an object from two different positions, the respective focal lengths can be compared to determine the dimensions of the object. A more advanced method uses laser beams and sensors to calculate these lengths based on the speed of the beam and the duration of the reflection from the surface. The

\footnotetext{
*Correspondence: almos99@freemail.hu
}

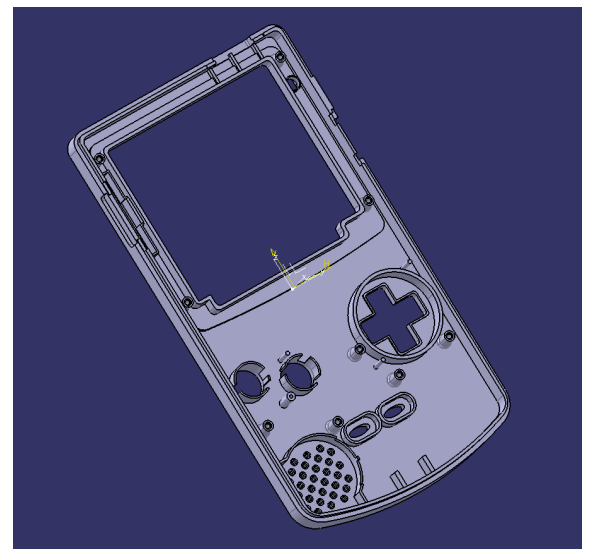

Figure 1: CAD model of the shell of a handheld game console.

current position of the device is also required [2]. None of these methods requires physical contact with the object.

Today, 3D scanning devices fit in our pockets: numerous smartphones and pieces of computer software are available that can create 3D models based on photos. Entry-level, free software typically offers cloud-based processing that may take days to finish. In order to access higher-end, engineering-level software, a subscription must be taken out, however, instant results are provided.

At least 40 photos are required to model the geometry - the more photos, the better the result. Photos should be taken from roughly equiangular positions on a circle with a $30-300 \mathrm{~cm}$ radius around the object in a static environment. The software overlaps the photos based on the surroundings of the target object (like pieces of a jigsaw puzzle), ultimately creating a three-dimensional replica of the environment with the 


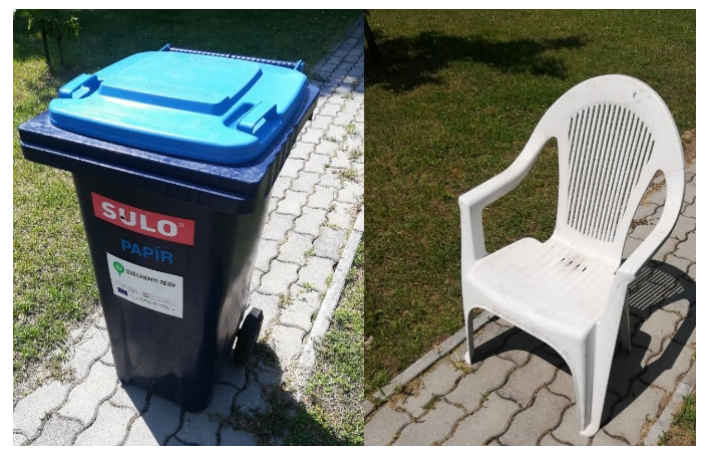

Figure 2: Rubbish bin and garden chair.

object in the middle.

This technology is applied in practice to map nature reserves [3], to investigate crime and accident scenes [4], as well as in medical and production technologies [5].

\section{Experiment}

The piece of software "Autodesk ReCap Photo" was used with an educational license to generate models during the experiment. The accuracy of their size, shape and ratio was evaluated, along with their potential practical applications. However, this strongly depends on the type of usage and an accuracy within a range of $1-2 \%$ is generally expected [6].

The photos were taken with a 16-megapixel smartphone camera. The software supports both landscape and portrait image orientations. Model generation is cloud-based, meaning powerful hardware is not required, however, a processing time of $20-70$ hours should be expected.

Models are generated by the aforementioned overlaying principle. The more photos there are, the more accurate the layering and, therefore, the model itself will be [7]. A total of 4 objects of different sizes and complexities were modelled. The experiments and their results are presented below.

The first objects that were attempted to be modelled were a plastic rubbish bin and a garden chair (Fig. 2).

Photos were taken from both sides and above. The photographic parameters used are summarized in Table 1. Since the photos were taken outdoors, a heightened level of attention was needed to include any moving objects (tree branches, cats, birds, etc.) in the photos as well as achieve consistent lighting and minimal shading or glistening off the surfaces. These distructions had a negative effect on the generated model. The results (cut and positioned) can be seen in Fig. 3 .

Table 1: Photographic parameters.

\begin{tabular}{lll}
\hline Object & $\begin{array}{l}\text { Distance } \\
\text { from camera }\end{array}$ & $\begin{array}{l}\text { Number } \\
\text { of photos }\end{array}$ \\
\hline rubbish bin & $120-150 \mathrm{~cm}$ & 49 \\
garden chair & $120-150 \mathrm{~cm}$ & 40 \\
\hline
\end{tabular}

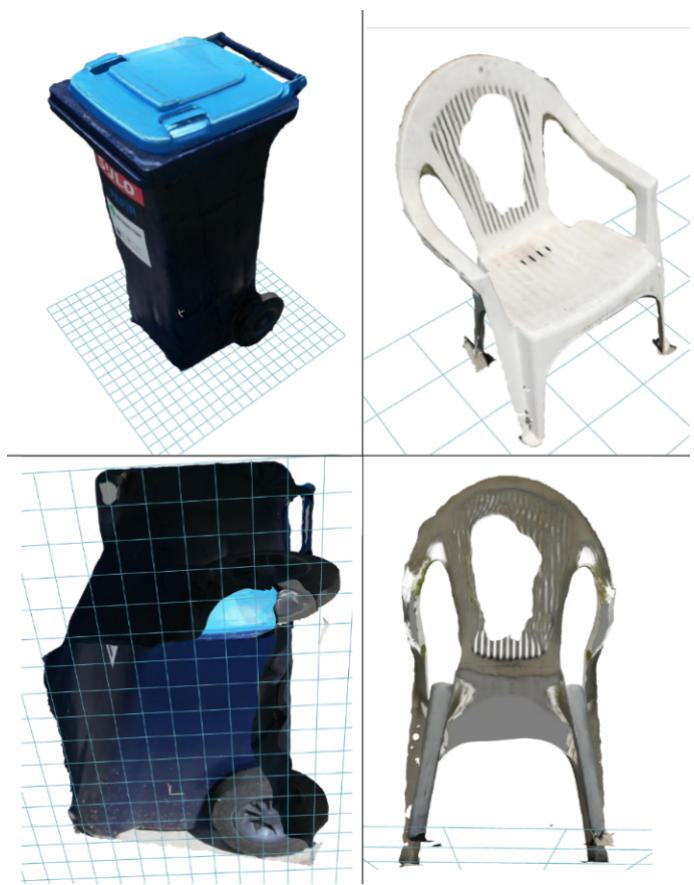

Figure 3: Models of the rubbish bin and garden chair.

The bottom parts of the models are visibly inaccurate and hollow since no information was present in the photos. The model of the rubbish bin is specifically inaccurate around the wheels and a minimal degree of waviness of the side panels can be observed. The model of the garden chair features one large and several smaller holes on the back, supposedly as a result of its thin structure, inappropriate lighting and shades.

Nevertheless, the fundamental shapes and details are present, significantly reducing the post-processing time to create a perfect model compared to manual measurements and modelling from scratch.

Although the models are highly detailed, a high level of accuracy and rational dimensions are also crucial for practical applications. A rectangular box was used to determine the accuracy of the software. The box along with its dimensions can be seen in Fig. 4.

Once the project has been processed, the model can

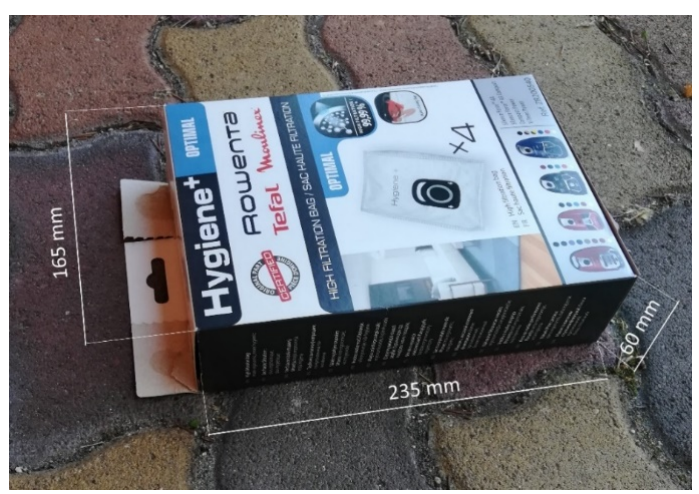

Figure 4: Rectangular box with dimensions used to validate the level of accuracy. 


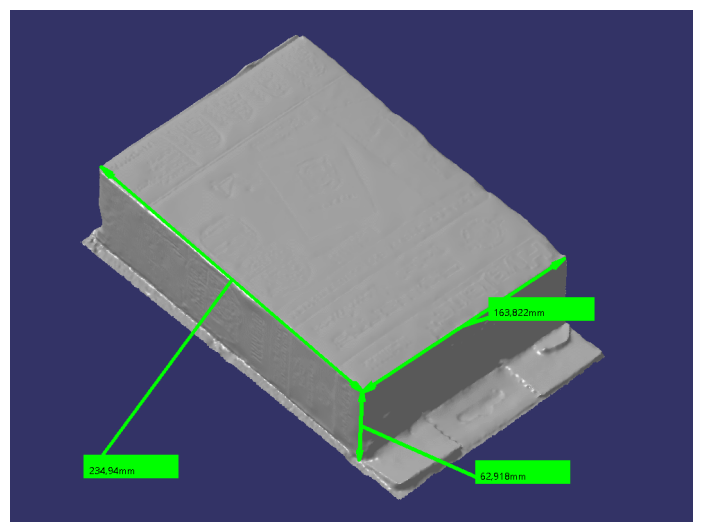

Figure 5: Generated dimensions.

be edited directly from ReCap Photo. It is possible to move, rotate or remove parts of the object. A dimension can be set manually between two arbitrary points, after which all the dimensions are calculated by the software proportionally. The model can then be exported in various polygon mesh formats and imported into various pieces of CAD software for post-processing. Dassault Systèmes' CATIA software was used to measure the generated dimensions. The results can be seen in Fig. 5 .

The comparison between the actual and generated dimensions is presented in Table 2 .

As can be seen in Table 2, the most significant deviation is $-4.863 \%$. However, this is the only dimension in connection with the deformed bottom surface, the geometry of which is inaccurate due to the photography. The deviation in the other two directions is under $1 \%$; this accuracy is acceptable in most branches of engineering. In the case of a significant practical application, a higher-quality camera should be used to achieve better photographs as well as a pedestal to prevent any surfaces from touching the ground, thereby achieving an exponentially higher-quality model.

As a final project, an attempt was made to model an automobile and a motorcycle. These objects include extensive, complex geometries and irregular, glistening, transparent surfaces, which pose a challenge for the software. Photos of both vehicles and the generated models can be seen in Figs. 6 and 7, respectively.

Several faults can be observed in the models. The roof of the car is inaccurate due to the lack of top view photos. The windows are not smooth and its surface is wavy in certain areas as if it was "molten". This can also

Table 2: Comparison between the actual and generated dimensions.

\begin{tabular}{llll}
\hline $\begin{array}{l}\text { Actual } \\
\text { dimensions }\end{array}$ & $\begin{array}{l}\text { Generated } \\
\text { dimensions }\end{array}$ & $\begin{array}{l}\text { Difference } \\
(\mathrm{mm})\end{array}$ & $\begin{array}{l}\text { Difference } \\
(\%)\end{array}$ \\
\hline $235 \mathrm{~mm}$ & $234.94 \mathrm{~mm}$ & $0.06 \mathrm{~mm}$ & $0.0256 \%$ \\
$165 \mathrm{~mm}$ & $163.822 \mathrm{~mm}$ & $1.178 \mathrm{~mm}$ & $0.714 \%$ \\
$60 \mathrm{~mm}$ & $62.918 \mathrm{~mm}$ & $2.918 \mathrm{~mm}$ & $-4.863 \%$ \\
\hline
\end{tabular}

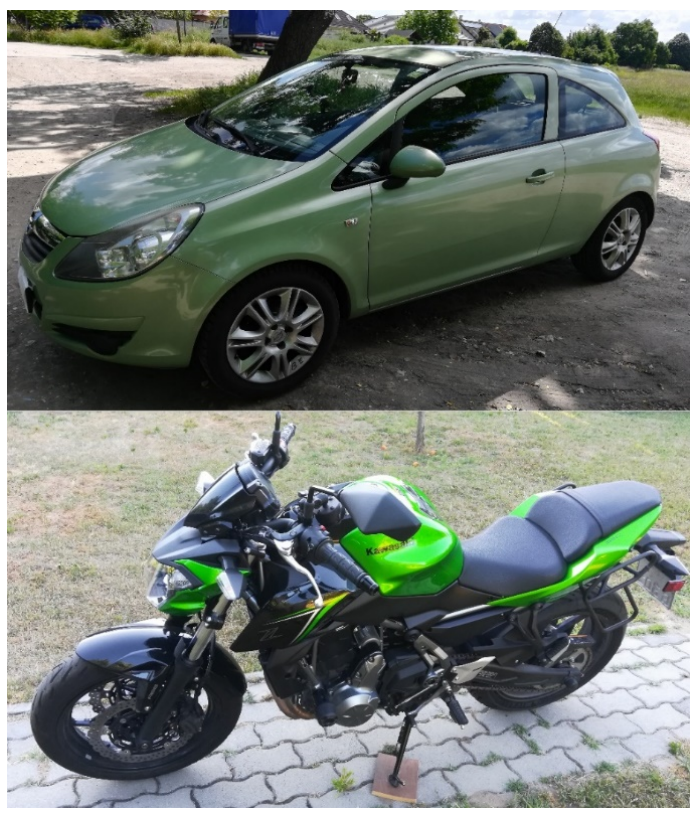

Figure 6: Photographs of the automobile and the motorcycle.
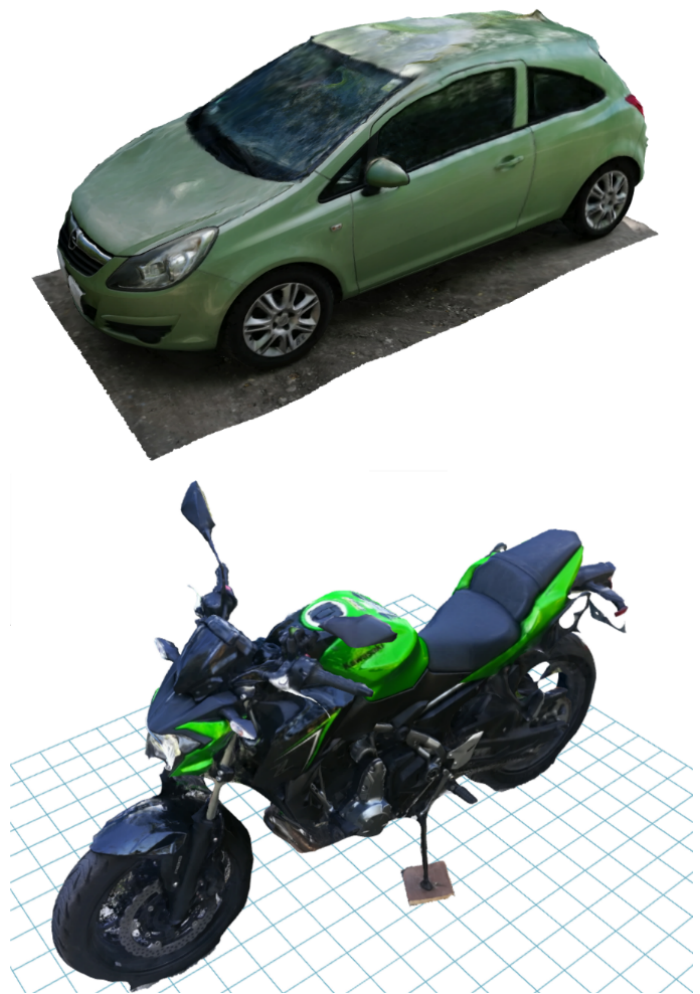

Figure 7: Generated models of the automobile and the motorcycle.

be seen on the motorcycle, along with the inaccuracy of the tires, especially on areas close to the ground. Despite these faults, the main shapes and ratios are maintained. Modifying these models by post-processing is more straightforward and quicker than creating one from scratch. 


\section{Conclusion}

With the development of 3D printing, an increased emphasis is being placed on reverse-engineering technologies to quickly and efficiently copy existing geometries either for the purpose of recreation or simulations. Photo-based 3D scanning software play a crucial role in simplifying and drastically shortening this process, offering an economical, quick and space-saving alternative to traditional 3D scanning devices. The method can be used outside of engineering applications, e.g., in medicine to create custom implants and prostheses or in accident scene investigation by running simulations on the modelled wreckage. The study shows that the accuracy of ReCap Photo is sufficient for these applications, offering expert solutions in various fields.

\section{REFERENCES}

[1] Rekoff, M.G.: On Reverse Engineering, IEEE Trans. Syst. Man Cybern., 1985, 15(2), 244-252 DOI: 10.1109/TSMC.1985.6313354

[2] Edl, H.; Mizerák, M.; Trojan, J.: 3D Laser Scanners: History and Applications. Acta Simulatio, 2018, 4(4), 1-5 DOI: 10.22306/asim.v4i4.54

[3] Remondino, F.: Heritage Recording and 3D Modeling with Photogrammetry and 3D Scanning.
Remote Sensing, 2011, 3(6), 1104-1138 DOI: 10.3390/rs3061104

[4] Barazzetti, L.; Sala, R., Scaioni, M.; Cattaneo, C.; Gibelli, D.; Giussani, A.; Poppa, P.; Roncoroni, F.; Vandone, A.: 3D scanning and imaging for quick documentation of crime and accident scenes. Sensors, and Command, Control, Communications, and Intelligence (C3I) Technologies for Homeland Security and Homeland Defense XI, Ed. Carapezza, E.M., 2012, 8359, 208-221 DOI: 10.1117/12.920728

[5] Ciobanu, O.; Xu, W.; Ciobanu, G.: The Use of 3D Scanning and Rapid Prototyping in Medical Engineering. Fiability \& Durability, 2013, 1, 241247 ISSN: 1844-640X

[6] Mankovits, T.; Szabó, T.; Kocsis, I.; Páczelt, I.: Optimization of the shape of axi-symmetric rubber bumpers. Stroj. Vestn.-J. Mech. E., 2014, 60(1), 6171 DOI: $10.5545 /$ sv-jme.2013.1315

[7] Fedorko, G.; Stanova, E.; Molnar, V.; Husakova, N.; Kmet, S.: Computer modelling and finite element analysis of spiral triangular strands. Adv. Eng. Softw., 2014, 73, 11-21 DOI: 10.1016/j.advengsoft.2014.02.004 